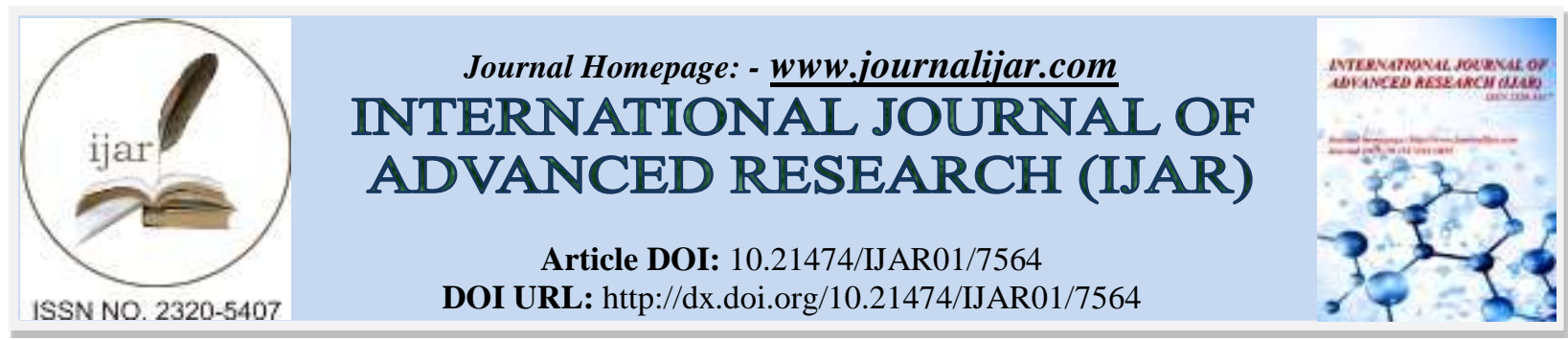

RESEARCH ARTICLE

\title{
RIGHT HEART REMODELING AND ITS EVALUATION IN THE CONTEMPORARY GUIDELINES OF ARTERIAL HYPERTENSION MANAGEMENT.
}

\section{Ana Minashvili MD ${ }^{1}$ and Ann Rekhviashvili MD, $\mathbf{P h D}^{2}$.}

1. PhD student of the Ivane Javakhishvili Tbilisi State University; Doctor-Cardiologist at the G. Chafidze Heart Center. Tbilisi, Georgia.

2. Associate Professor at the Petre Shotadze Tbilisi Medical Academy. Chief of the Arterial Hypertension and Vascular Study Center at the Archangel St. Michael Multiprofile Clinical Hospital. Tbilisi, Georgia.

\section{Manuscript Info}

(.........................

Manuscript History

Received: 14 June 2018

Final Accepted: 16 July 2018

Published: August 2018

Keywords:-

Arterial hypertension * right ventricular remodeling * right heart function.

\begin{abstract}
Left ventricular structural and functional changes in patients with arterial hypertension were well established. However, the influence of arterial hypertension on right ventricular remodeling is still under investigation. The systematic assessment of right heart function, right ventricular size and geometry was lacking due to the underestimation of its meaning, complex shape and technical issues. Development of cardiovascular medicine, particularly improvement of cardiac imaging techniques, has revealed that the right ventricle has a vital role in a global cardiac function, cardiovascular and total morbidity and mortality. Clinical studies revealed an increased pulmonary vascular resistance in patients with arterial hypertension. Some studies showed that even early abnormal increase in arterial blood pressure may lead to the changes in right ventricular pressures, volumes and ejection fraction. It was confirmed by several studies, that influence of arterial hypertension is on the whole heart, namely hypertrophy of both ventricles mostly coexist, which strengthens the concept that the right and left ventricles physiologically represent a single functional unit. Hence, this phenotype can be considered as a very high cardiovascular risk profile. In spite of the clinical studies showed that the right ventricle plays an important role in the morbidity and mortality of patients with arterial hypertension, the term "right heart" is not even mentioned in the world leading guidelines. Consequently, the purpose of this review article is to provide the overview of the current knowledge regarding the relationship between arterial hypertension and right ventricular structure, function, and its prognostic meaning.
\end{abstract}

Copy Right, IJAR, 2018,. All rights reserved.

\section{Introduction:-}

During the decades an enormous attention was given to the evaluation of the left heart in patients with arterial hypertension. Left ventricle's geometry and function has been studied extensively. Hence, normal values of left ventricular (LV) dimensions, volumes, mass variability, functional characteristics were well established. Therefore, the importance of right ventricle assessment and clinical meaning of its remodeling and functional disturbances has long been neglected. 
Sir William Harvey was the first who described the importance of right ventricle and its function in his treatise "De Motu Cordis" in 1616 year. Therefore, for many years followed, study of the RV was overshadowed. Only in the first half of the $20^{\text {th }}$ century evaluation of the RV started again and only a small group of investigators were intrigued by the hypothesis that human circulation could function adequately without RV contractile function (Goldstein, 2005; Haddad et al., 2008). From 1950 to late 1970s studying of right heart became relatively important and significance of RV function has been proposed in heart failure, RV myocardial infarction, congenital heart disease and pulmonary hypertension.

The systematic assessment of right heart function, RV size, geometry and function was lacking due to the underestimation of its meaning, complex shape and technical issues. The development of cardiovascular medicine, particularly the improvement of cardiac imaging techniques, has revealed that the RV has a vital role in global cardiac function, cardiovascular and total morbidity, and mortality. Not only in diseases that predominantly involve pulmonary circulation, such as pulmonary hypertension and congenital heart diseases, but also in conditions that primarily affect the left ventricle (LV), such as heart failure and mitral and aortic valve diseases (Akintunde et al., 2010). First precise and relatively full recommendations regarding RV assessment was given in the "Guidelines for the Echocardiographic Assessment of the Right Heart in Adults" (Rudski et al., 2010). It should be mentioned that the basics of RV dimensions and function were partly and quite superficially described earlier in the American Society of Echocardiography and European Association of Echocardiography recommendations for chamber quantification published in 2005, which mainly focused on the left heart assessment (Lang et al., 2005). Hence, there is a need for further improvement of diagnostic methodology as well as standardization and unification of the RV echocardiographic examination in the world. Consequently, the purpose of this review article is to provide the overview of the current knowledge regarding the relationship between essential hypertension and RV structure, function, and its prognostic meaning.

\section{Macro- and microscopic anatomy of the right ventricle}

The RV is the most anteriorly located cardiac chamber in a healthy, normal heart and lies immediately behind the sternum. It is delimited by the tricuspid and pulmonary valves. It was suggested by Goor and Lillehei in 1975, that the RV has 3 components; namely, (1) the inlet, which consists of the tricuspid valve, chordae tendineae, and papillary muscles; (2) the trabeculated apical myocardium; and (3) the infundibulum, or conus, which corresponds to the smooth myocardial outflow region (Haddad et al., 2008).

Some investigators prefer dividing of the RV into anterior, lateral, and inferior walls, as well as basal, mid, and apical sections. In the RV there are presented three muscular bands, namely the parietal band, the septomarginal band, and the moderator band. The septomarginal band extends inferiorly and becomes continuous with the moderator band, which attaches to the anterior papillary muscle. Unique characteristic feature of the RV is the presence of a ventriculoinfundibular fold that separates the tricuspid and pulmonary valves, while mitral and aortic valves are in a fibrous continuity in the left ventricle.

RV has a complex shape. In normal conditions, the septum is concave toward the LV in both systole and diastole. In a healthy heart, the RV is crescent shaped viewed in a cross section and triangular shaped when viewed from the side. It should be mentioned that the shape of the RV may be influenced by the position of the interventricular septum. In adults the volume of the RV is larger than the volume of the LV, whereas RV mass is approximately one sixth that of the LV (Lorenz et al., 1999).

The ventricles are composed of a multiple muscle layers that form a 3-dimensional (3D) network of fibers. According to the Ho and Nihoyannopoulos description, RV wall is mainly composed of superficial and deep muscle layers (Ho and Nihoyannopoulos, 2006). The fibers of the superficial layer of the RV are arranged more or less circumferentially and the deep muscle fibers are longitudinally aligned base to apex.

The position of the RV in the chest makes the RV assessment challenging for a conventional imaging technique echocardiography. Cardiac magnetic resonance and cardiac computer tomography are more accurate and provide better insight, but their availability and cost still represent the major limitation to their usage in everyday clinical practice. The introduction of three-dimensional echocardiography (3DE) and speckle tracking imaging has significantly changed the approach to the RV evaluation providing the information regarding RV size, shape, function, and mechanics (Tadic, 2015). 


\section{The impact of $L V$ changes on $R V$ mechanics}

The importance of LV changes, namely abnormalities in geometry and its hypertrophy on cardiovascular morbidity and mortality were previously confirmed in many observational and longitudinal studies, as well as in metaanalyses. Nowadays many authors prefer to use new classification of LV hypertrophy dependent on the LV geometry, namely dilated and non-dilated forms of hypertrophy instead of concentric and eccentric forms of LV hypertrophy (Khouri et al., 2010). Supporters of new classification suggest that it can improve mortality risk stratification in a general population. There are many hypotheses that try to find the connection between LV changes and cardiovascular risk, but most of them are based only on the influence of increased LV mass on morbidity and mortality.

The influence of LV changes in geometry and mass on RV remodeling is not studied well yet and hence is intriguing. According to the study data presented by Karaye et al. (2012), hypertensive patients with eccentric LV hypertrophy had the most dilated RV and lowest RV systolic function assessed by tissue Doppler and TAPSE. The authors did not find any significant difference between the hypertensive patients with normal geometry, concentric remodeling, and concentric hypertrophy.

\section{RV Structure and Function in Arterial Hypertension}

In comparison with the left heart performance during different cardio-vascular diseases, the right ventricular performance has received only scant attention. Potential dysfunction of RV was mostly studied in patients with acute and chronic coronary artery disease and lung diseases (Polak et al., 1983; Johnson et al., 1979; Ferlinz et al. 1976). Very little is known about the RV performance while arterial hypertension. It has been proven by Stool et al., (1974), Moulopoulos et al., (1965), Kelly et al., (1971), Taylor et al., (1967), Bemis (1974) that distortion of the normal geometry of one of the two ventricles may influence other ventricle's performance. Atkins et al. (1977) showed an increased pulmonary vascular resistance in patients with systemic hypertension. Ferlinz (1980) was the first, who hypothesized that chronic left ventricular pressure overload in patients with essential hypertension may impact on RV function. Ferlinz and his colleagues demonstrated that right-sided circulation was vulnerable to the effects of systemic hypertension; namely, in comparison with normotensive subjects, hypertensive individuals had an increased right-sided heart pressures and volumes than the controls with normal blood pressure. Study authors showed an augmented RV afterload due to the increased pulmonary pressures in the patients with arterial hypertension, which leads to the larger end systolic volume index (ESVI) and lower ejection fraction (EF). Thus, a stressed LV may adversely affect RV performance and cause changes in the volume-pressure characteristics of RV. Study showed that even early abnormal increase in arterial blood pressure may lead to the changes in RV pressures, volumes and EF. Hence, influence of AH is on the whole heart, which strengthens the concept that the right and left ventricles physiologically represent a single functional unit.

Decades ago it was postulated that essential hypertension may cause hypertrophy of the left ventricular septum, which could be encroached into the right ventricle and cause the Bernheim's syndrome, which consists of symptoms and signs of pronounced right ventricular cardiac failure (venous hypertension, hepatomegaly and edema) appearing in the absence of dyspnea and orthopnea.

Myslinski at al., (1998) studied RV structure and function in 59 patients with untreated, uncontrolled mild to moderate essential systemic hypertension via echocardiographic assessment of heart. None of the patients had symptoms of congestive heart failure, ischaemic heart disease, lung disorders or endocrinopathies. Study results confirmed an existence of the impaired diastolic filling of both left and right ventricles. Study authors found out a free wall thickening of a RV and interventricular septum hypertrophy. Hence they concluded that RV can be considered as a major factor influencing RV performance.

Akintunde et al. (2010) and his colleagues hypothesized that the spectrum of changes in structure, function and shape of the left ventricle while arterial hypertension should have an effect on the structure and function of the right ventricle. Hence, aim of the study was to evaluate if any possible morphological and/or functional changes might occur in the right ventricle in subjects with systemic hypertension. 100 patients with arterial hypertension and 50 age and gender-matched normotensive control subjects were involved in a study. Echocardiographic method was used for description of the impact of hypertension induced left ventricular pressure overload and hypertrophy on right ventricular morphology and function. According to the study results researchers concluded that arterial hypertension is associated with the right ventricular morphological and functional abnormalities. AH affects the diastolic function of left ventricle and these changes are accompanied by similar changes in the right ventricle; namely, there were 
revealed changes in the diastolic wave velocities, right ventricular wall dimensions and internal chamber dimensions.

Cuspidi et al. (2009) examined 330 untreated uncomplicated essential hypertensive patients from out-patient hypertension clinic. They found out that more than one-fifth of the patients seen in a specialist setting have RV hypertrophy associated with left ventricle hypertrophy i.e. biventricular hypertrophy. Hence, this phenotype can be considered as a very high cardiovascular risk profile.

Using steady-state free precession cardiac MRI technique Todiere et al. (2011) and Lu et al. (2015) evaluated association of mild to moderate uncomplicated hypertension with abnormal right ventricular structure and function and its relationship with the changes in the left ventricle. Analyzes of the study data showed that systemic hypertension is in association with concentric right ventricular remodeling and impaired diastolic function; hence, unstressed right ventricle is not immune to the effects of systemic hypertension. Study investigators concluded that structural and functional adaptation of right ventricle to the systemic hypertension is parallel to the homologous modifications of the left ventricle.

Cuspidi et al. (2013) performed an analyze of the literature, namely 13 studies including 1290 hypertensive and 259 normotensive control subjects, to reveal right ventricular structural changes associated to systemic hypertension and concluded that RV hypertrophy is a common cardiac phenotype in systemic hypertension.

Pedrinelli et al. (2010) investigated 98 never treated, non-obese patients with blood pressure values varying from the optimal to the mild hypertension range for analyzing the relationship between increasing systemic blood pressure and right ventricular function. They confirmed that RV diastolic and systolic function deteriorates in response to slightly increased systemic blood pressure and is parallel to the LV changes. Authors of the study concluded biventricular interdependence caused by interventricular septum remodeling. Hanboly et al. (2015) showed similar results; namely, right ventriclular diastolic functional deterioration in response to slightly increased blood pressure in systemic circulation. According to the study investigators, right ventricular diastolic dysfunction can be considered as an early clue to hypertensive heart disease.

Measures of the right and left ventricular thickness were studied in 90 autopsy materials by Pessoa et al. (2012) and in 372 autopsy protocols by Kerimkulova et al. (2011). All the examined subjects had a history of essential hypertension. The studies confirmed the relationship of RV and LV hypertrophy, which suggest that there are similar pathogenic factors involved in the development of bilateral hypertrophy.

Scientists have shown not only changes in RV function and structure, but also a significant deterioration of RV mechanics, namely longitudinal deformation in patients with essential hypertension (Tadic et al., 2014, 2015, 2016, 2018). They revealed the association between RV longitudinal strain and functional capacity in hypertensive patients (Tadic and Ivanovic, 2014). According to the Lu et al. (2015), RV longitudinal strain should be considered as the strongest independent predictor for RV functional changes. Scientists showed strong relationship between RV longitudinal strain and cardiovascular mortality and morbidity in a wide range of cardiovascular conditions [Peyrou et al., 2017; D’ndrea et al. 2016).

The cross-sectional study of 186 untreated subjects with masked hypertension, normotension and sustained hypertension showed significantly lower RV global and free-wall longitudinal strains in patients with masked and sustained hypertension. Hence, study confirmed significant change of the RV structure, function, and deformation in subjects with masked and sustained hypertension (Tadic et al., 2016).

In 2009 a literature search was published by Tehrani and his colleagues (2009), where they were looking for the interrelationships between left and right ventricular diastolic dysfunction and diastolic heart failure. According to their conclusion, the timeline and progression of diastolic dysfunction to diastolic heart failure have not been well established yet and further investigation is needed.

Right heart assessment in contemporary guidelines of arterial hypertension management

In spite of the clinical studies showed that the right ventricle plays an important role in the morbidity and mortality of patients with arterial hypertension and the right ventricular function is strongly associated with clinical outcomes, the term "right heart", its remodeling and assessment necessities are not even mentioned in the world leading 
guidelines of the arterial hypertension management, like the "Report of the American College of Cardiology and American Heart Association Task Force on Clinical Practice Guidelines" (Aronow et al. 2017), published in the 2017 as well as in the "Australian guideline for the diagnosis and management of hypertension in adults" (Anderson et al. 2017), published in 2016, "Canadian guidelines for diagnosis, risk assessment, prevention, and treatment of hypertension in adults" published in 2017 and 2018 (Leung et al., 2017; Nerenberg et al. 2018), "ESH/ESC Guidelines for the management of arterial hypertension" (Mancia et al., 2013) published in 2013, "Japanese Society of Hypertension Guidelines for the Management of Hypertension" (Shimamoto et al. 2014) published in 2014, "Clinical management of primary hypertension in adults" published by the commission of the National Institute of Health and Clinical Excellence in 2011 (NICE 2011).

\section{Conclusion:-}

As this finding is based on a limited number of cross-sectional studies including small population samples, further investigations are needed to determine the clinical utility and prognostic value of this phenotype in clinical practice.

Clinical studies consistently indicate that RV hypertrophy is a common cardiac phenotype in patients with arterial hypertension. Interrelationship between left and right ventricles while arterial hypertension is well confirmed in many studies. But because of its complex shape and marked load dependence, the study of the RV remains challenging. Therefore, the question "What came first, the chicken or the egg" is still actual; namely, whether right heart changes occur early, at the same time as left heart changes due to the interdependence of the two structures, or whether it is a secondary phenomenon possibly related to pulmonary vascular changes remains to be proven by further studies involving patients with arterial hypertension.

Many studies, where were investigated influence of arterial hypertension on RV geometry and function and its prognostic meaning, were based on a cross-sectional studies including small population samples; hence, further investigations are needed to determine the clinical utility and prognostic value of this phenotype in clinical practice. For practitioners involved in hypertension management would be very useful and interesting to see professional discussion in contemporary guidelines of arterial hypertension regarding changes of RV function and geometry in arterial hypertension, its assessment and management options, known and unknown issues.

\section{Literature:-}

1. Akintunde, A., Akinwusi, P., Familoni, O. and Opadijo, O. (2010): Effect of systemic hypertension on right ventricular morphology and function: an echocardiographic study. Cardiovasc. J. Afr., 21: 252-256.

2. Anderson, C., Arnolda, L., Cowley, D., Dowden, J., Gabb, G., Golledge J., Hankey, G., Howes, F., Leckie, L., Mangoni, A., Perkovic, V., Schlaich, M. and Zwar N. (2017): Guideline for the diagnosis and management of hypertension in adults. National Heart foundation of Australia. Hypertension. 00:e000-e000.

3. Atkins, Z., Mitchell, H., Pettinger, W. (1977): Increased pulmonary vascular resistance with systemic hypertension. Effect of monoxidil and other antihypertensive agents. Am. J. Cardiol. 39:802-807.

4. Bemis, C.E., Serur, J.R., Borkenhagen, D., Sonnenblick, E., Urschel, C.W. (1974): Influence of right ventricular filling pressure on left ventricular pressure and dimension. Circ. Res. 34:498-504.

5. Cuspidi, C., Negri, F., Giudici, V., Valerio, C., Meani, S., Sala, C., Esposito, A., Masaidi, M., Zanchetti, A. and Mancia, G. (2009): Prevalence and clinical correlates of right ventricular hypertrophy in essential hypertension. J. Hypertens. 27(4):854-860.

6. Cuspidi, C., Sala, C., Muiesan, M., De Luca, N., Schillaci, G. (2013): Right ventricular hypertrophy in systemic hypertension: an updated review of clinical studies. J. Hypertens. 31:858-865.

7. D'ndrea A, Stanziola A, D'lto M, Di Palma, E., Martino, M., Scarafile, R., Molino, A., Rea, G., Maglione, M., Calabrò, R., Russo, M.G., Bossone. E. and Saggar, R. (2016): Right ventricular strain: an independent predictor of survival in idiopathic pulmonary fibrosis. Int. J. Cardiol. 222:908-910.

8. Ferlinz, J. (1980): Right ventricular performance in patients with essential hypertension. Circulation. 61(1):156-162.

9. Ferlinz, J., Del Vicario, M., Gorlin, R. (1976): Incidence of right ventricular asynergy in patients with coronary artery disease. Am. J. Cardiol. 38:557-563.

10. Goldstein, J. (2005): The right ventricle: what's right and what's wrong. Coronary Artery Diseases. 16:1-3.

11. Haddad, F., Hunt, S., Rosenthal, D. and Murphy, D. (2008): Right Ventricular Function in Cardiovascular Disease, Part I. Anatomy, Physiology, Aging, and Functional Assessment of the Right Ventricle. Circulation. 117:1436-1448. 
12. Hanboly, N.H. (2015): Right ventricle diastolic function in high-normal and mildly elevated arterial blood pressure. Ulutas Medical Journal. 1(4):100-103.

13. Ho, S.Y., Nihoyannopoulos, P. (2006): Anatomy, echocardiography, and normal right ventricular dimensions. Heart. 92(suppl 1):i2-i13.

14. Johnson, L., McCarthy, D., Sciacca, R. and Cannon, P. (1979): Right ventricular ejection fraction during exercise in patients with coronary artery disease. Circulation 58(suppl II):11-61.

15. Karaye, K.M., Sai'du, H., Shehu, M.N. (2012): Right ventricular dysfunction in a hypertensive population stratified by patterns of left ventricular geometry. Cardiovasc. J. Afr. 23:478-482.

16. Kelly, D.T., Spotnitz, H.M., Beiser, G.D., Pierce, J.E., Epstein, S.E. (1971): Effects of chronic right ventricular volume and pressure loading on left ventricular performance. Circulation. 44:403-412.

17. Kerimkulova, A. (2011): Research of wall thickness of left and right ventricles in patients with arterial hypertension basing on postmortem examination. Vestnik of Novgorod State University. 62:64-66.

18. Khouri, M.G., Peshock, R.M., Ayers, C.R., de Lemos, J.A., Drazner, M.H. (2010): A 4-tiered classification of left ventricular hypertrophy based on left ventricular geometry: the Dallas heart study. Circ. Cardiovasc Imaging 3:164-171.

19. Lang, R.M., Bierig, M., Devereux, R., Flachskampf, F.A., Foster, E., Pellikka, P.A., Picard, M.H., Roman, M.J., Seward, J., Shanewise, J.S., Solomon, S.D., Spencer, K.T., St John Sutton, M., Stewart, W.J. (2005): Recommendations for chamber quantification: a report from the American Society of Echocardiography's Guidelines and Standards Committee and the Chamber Quantification Writing Group, developed in conjunction with the European Association of Echocardiography, a branch of the European Society of Cardiology. J. Am. Soc. Echocard. 18:1440-63.

20. Leung, A., Daskalopoulou, S., Dasgupta, K., McBrien, K., Butalia, S., Zarnke, K.B., Nerenberg, K., Harris, K.C., Nakhla, M., Cloutier, L., Gelfer, M., Lamarre-Cliche, M., Milot, A et al. (2017): Hypertension Canada's 2017 Guidelines for Diagnosis, Risk Assessment, Prevention, and Treatment of Hypertension in Adults. Canadian Journal of Cardiology 33:557-576.

21. Lorenz, C.H., Walker, E.S., Morgan, V.L., Klein, S.S., Graham, T.P. Jr. (1999): Normal human right and left ventricular mass, systolic function, and gender differences by cine magnetic resonance imaging. J. Cardiovasc. Magn. Reson. 1:7-21.

22. Lu, K,J., Chen, J.X., Profitis, K., Kearney, L.G., DeSilva, D., Smith, G., Ord, M., Harberts, S., Calafiore, P., Jones, E., Srivastava, P.M. (2015): Right ventricular global longitudinal strain is an independent predictor of right ventricular function: a multimodality study of cardiac magnetic resonance imaging, real time threedimensional echocardiography and speckle tracking echocardiography. Echocardiography. 32:966-974.

23. Mancia, G., Fagard, R., Narkiewicz, K. (2013): 2013 ESH/ESC Guidelines for the management of arterial hypertension. The Task Force for the management of arterial hypertension of the European Society of Hypertension (ESH) and of the European Society of Cardiology (ESC). Journal of Hypertension. 31:12811357.

24. Moulopoulos, S., Sarcas, A., Stamatelopoulos, S., Arelias, E. (1965): Left ventricular performance during bypass or distension of the right ventricle. Circ. Res. 17:484-491.

25. Myslinski, W., Mosiewicz, J., Ryczak, E., Barud, W., Biłan, A., Palusiński, R. and Hanzlik, J. (1998): Right ventricular function in systemic hypertension. J. Hum. Hyp. 12:149-155.

26. Nerenberg, K., Zarnke, K., Leung, T. (2018): A. Hypertension Canada's 2018 Guidelines for Diagnosis, Risk Assessment, Prevention, and Treatment of Hypertension in Adults and Children. Canadian Journal of Cardiology. 34:506-525.

27. Pedrinelli, R., Canale, M.L., Giannini, C., Talini, E., Penno, G., Dell'Omo, G. and Di Bello, V. (2010): Right ventricular dysfunction in early systemic hypertension: a tissue Doppler imaging study in patients with highnormal and mildly increased arterial blood pressure. J. Hypertens. 28:615-621.

28. Pessoa, M., José, R., Tavares, M. and Modesto, M. (2012): Left and right ventricular hypertrophy at autopsy of hypertensive individuals. Rev. Assoc. Med. Bras. 58(1):41-47.

29. Peyrou, J., Chauvel, C., Pathak, A., Simon, M., Dehant, P. and Abergel, E. (2017): Preoperative right ventricular dysfunction is a strong predictor of 3 years survival after cardiac surgery. Clin. Res. Cardiol. 106:734-742.

30. Polak, J., Holman, L., Wynne, J., Colucci, W. (1983): Right ventricular ejection fraction: An indicator of increased mortality in patients with congestive heart failure associated with coronary artery disease. J. Am. Coll. Card. 2(2):217-224.

31. Rudski L.G., Lai W.W., Afilalo J, Handschumacher, H.L., Chandrasekaran, K., Solomon S.D., Louie, E.K., Schiller, N.B. (2010): Guidelines for the Echocardiographic Assessment of the Right Heart in Adults: A 
Report from the American Society of Echocardiography. Endorsed by the European Association of Echocardiography, a registered branch of the European Society of Cardiology, and the Canadian Society of Echocardiography. Journal of the American Society of Echocardiography. 23:685-713.

32. Shimamoto, K., Ando, K., Fujita, T. (2014): The Japanese Society of Hypertension Guidelines for the Management of Hypertension (JSH 2014). Hypertension Research. 37:253-392.

33. Stool, E., Mullins, C., Leshin, S. and Mitchell, J. (1974): Dimensional changes of the left ventricle during acute pulmonary arterial hypertension in dogs. Am. J. Cardiol. 33:868.

34. Tadic M. (2015): Multimodality evaluation of the right ventricle: an updated review. Clin Cardiol. 38:770-776.

35. Tadic, M., Cuspidi, C., Bombelli, M. and Grassi, G. (2018): Right heart remodeling induced by arterial hypertension: Could strain assessment be helpful? J. Clin. Hypertens. 20:400-407.

36. Tadic, M., Cuspidi, C., Pencic, B., Sljivic, A., Ivanovic, B., Neskovic, A., Scepanovic, R. and Celic, V. (2014): High-normal blood pressure impacts the right heart mechanics: a three-dimensional echocardiography and twodimensional speckle tracking imaging study. Blood Press Monit. 19:145-152.

37. Tadic, M., Cuspidi, C., Pencic, B., Jozika, L. and Celic, V. (2015): Relationship between right ventricular remodeling and heart rate variability in arterial hypertension. J. Hypertens. 33:1090-1097.

38. Tadic, M., Cuspidi, C., Vukomanovic, V. (2016): The influence of masked hypertension on the right ventricle: is everything really masked? Journal of American Society of Hypertension. 10(4):318-324.

39. Tadic, M., Ivanovic, B. (2014): Why is functional capacity decreased in hypertensive patients? From mechanisms to clinical studies. J. Cardiovasc. Med. (Hagerstown). 15:447-455.

40. Taylor, R., Covell, J., Sonnenblick, E. and Ross, J. (1967): Dependence of ventricular distensibility on filling of the opposite ventricle. Am. J. Physiol. 213:711-718.

41. Tehrani, F., Phan, A., Schwarz, E. (2009): Left and right ventricular diastolic dysfunction and diastolic heart failure: does one lead to the other? J. Geriatr. Cardiol. 6:3-10.

42. Todiere G, Neglia D, Ghione S, Fommei, E., Capozza, P., Guarini, G., Dell'Omo, G., Aquaro, G.D., Marzilli, M., Lombardi, M.,Camici, P. and Pedrinelli, R. (2011): Right ventricular remodelling in systemic hypertension: a cardiac MRI study. Heart. 97:1257-1261.

43. The clinical management of primary hypertension in adults. Commissioned by the National Institute for Health and Clinical Excellence. National Clinical Guideline Center 2011 (NICE 2011).

44. Aronow, W., Ovbiagele, B., Casey, D.E. Jr., Smith, S.C. Jr., Collins, K.J., Spencer, C.C., Himmelfarb, C.D., Dennison C., Stafford, R., DePalma, S.M., Taler, S.J., Gidding, S., Thomas, R.J., Jamerson, K.A., Williams, Sr, K.A., Jones, D.A., Williamson, J.D., MacLaughlin, E.J., Wright, Jr, J.T., Muntner, P. (2017): 2017 ACC/AHA/AAPA/ABC/ ACPM/AGS/APhA/ASH/ASPC/NMA/PCNA Guideline for the Prevention, Detection, Evaluation, and Management of High Blood Pressure in Adults. A Report of the American College of Cardiology/American Heart Association Task Force on Clinical Practice Guidelines. (2017). Hypertension. 00:e1-e481. 